\title{
Investigation of Target Minimum and Maximum Dosimetric Criteria for the Evaluation of Standardized Radiotherapy Plan
}

\section{-Target Minimum and Maximum Evaluation}

\author{
Jialu Yu1 ${ }^{1}$ Huazhi Geng1, Yutao Gong1, Mitchell Machtay², \\ Himanshu R. Lukka ${ }^{3}$, Zhongxing Liao ${ }^{4}$, Ying Xiao' ${ }^{1}$, Wei Zou ${ }^{1 *}$ \\ ${ }^{1}$ Department of Radiation Oncology, University of Pennsylvania, Philadelphia, USA \\ ${ }^{2}$ Department of Radiation Oncology, University Hospitals of Cleveland, Cleveland, USA \\ ${ }^{3}$ Department of Radiation Oncology, Juravinski Cancer Centre at Hamilton Health Sciences, Hamilton, USA \\ ${ }^{4}$ Department of Radiation Oncology, M D Anderson Cancer Center, Houston, USA \\ Email: *wei.zou@pennmedicine.upenn.edu
}

How to cite this paper: Yu, J.L., Geng, H.Z., Gong, Y.T., Machtay, M., Lukka, H.R., Liao, Z.X., Xiao, Y. and Zou, W. (2020) Investigation of Target Minimum and Maximum Dosimetric Criteria for the Evaluation of Standardized Radiotherapy Plan. International Journal of Medical Physics, Clinical Engineering and Radiation Oncology, 9, 43-51.

https://doi.org/10.4236/ijmpcero.2020.92005

Received: January 20, 2020

Accepted: March 23, 2020

Published: March 26, 2020

Copyright $\odot 2020$ by author(s) and Scientific Research Publishing Inc. This work is licensed under the Creative Commons Attribution International License (CC BY 4.0).

http://creativecommons.org/licenses/by/4.0/

(c) (i) Open Access

\begin{abstract}
Purpose: Standardization of tumor dosimetric coverage is essential for the evaluation of radiotherapy treatment plan quality. National clinical trials network RTOG protocols include tumor target dosimetric criteria that specify the prescription dose and minimum and maximum dose $\left(D_{\min }\right.$ and $\left.D_{\max }\right)$ coverages. This study investigated the impact of various minimum and maximum dose definitions using tumor control probability (TCP) models. Methods and Materials: Three disease sites (head and neck, lung, and prostate) were studied using target volume dosimetric criteria from the RTOG 0920, 1308, and 0938 protocols. Simulated target dose-volume histograms (DVHs) of $D_{\min }$ and $D_{\max }$ were modeled using the protocol specifications. Published TCP models for the three disease sites were applied to the DVH curves. The effects of various dose definitions on TCP were studied. Results: While the prescription dose coverage was maintained, a $-3.7 \%$ TCP difference was observed for head and neck cancer when the target doses varied by $3.5 \%$ of the tumor volume from the point dose. For prostate and lung cancers, $-3.3 \%$ and $-2.2 \%$ TCP differences were observed, respectively. The TCPs for head and neck and prostate cancers were more negatively affected by deviations in the $D_{\min }$ than the TCP for lung cancer. The lung TCP increased to a greater extent with a change in the $D_{\max }$ compared with the head and neck and prostate TCPs. Conclusions: These results can be used to evaluate plan quality when the target dose only slightly deviates from the dosimetric criteria. When the
\end{abstract}


overall target prescription dose coverage is maintained, the $D_{\max }$ is recommended to be within 3\% of the target volume: $98 \%$ (for head and neck and prostate) and $97 \%$ (for lung) of the target volume, satisfying the $D_{\min }$ needed to maintain TCP variations at less than $2.1 \%$. Using $0.03 \mathrm{cc}$ instead of a point dose for $D_{\min }$ and $D_{\max }$ criteria minimally impacts TCPs.

\section{Keywords}

Rodiotherapy, Target Dosimetric Criteria

\section{Introduction}

The imaging and radiation oncology core (IROC) provides radiation therapy (RT) quality assurance services within the national clinical trials network [1] [2] [3] [4]. IROC service operations include site qualification, trial design support, credentialing, data management, and case review [1] [2] [3] [4].

Developing dosimetry review criteria is an important part of trial design support. Adding the planning tumor volume (PTV) to the dosimetry review criteria is necessary to achieve the tumor control probability (TCP) of the protocol [5]. PTV dosimetry review usually includes checking the prescription dose coverage of the PTV, as well as the minimum absorbed dose $\left(D_{\min }\right)$ and the maximum absorbed dose $\left(D_{\max }\right)$ in the PTV [6]. According to the ICRU report [7], the $D_{\min }$ might not be accurately determined since it is often located in a high-gradient region at the edge of the PTV, making it highly sensitive to the resolution of the calculation and the accuracy of either delineating the CTV or determining the PTV. Reporting of the $D_{\min }$ was replaced by the more accurate near-minimum absorbed dose, which is the dose covering 98\% of the PTV (D98\%) [7]. Similarly, the dose covering $2 \%$ of the PTV (D2\%) was recommended to be reported as the $D_{\max }$ [7]. However, alternative specifications of $D_{\min }$ and $D_{\max }$ are being used in different clinical settings, for example, the dose covering $99 \%$ of the PTV (D99\%) and the dose covering $1 \%$ of the PTV (D1\%), respectively. In RTOG protocols [6], $D_{\min }$ is usually reported as the dose covering the total PTV minus $0.03 \mathrm{cc}$ (Dvol $-0.03 \mathrm{cc}$ ), and $D_{\max }$ is usually reported as the dose covering $0.03 \mathrm{cc}$ of the PTV (D $0.03 \mathrm{cc})$.

The purpose of this study is to show the effects on TCP of different specifications of $D_{\min }$ and $D_{\max }$ of the target volume. The dosimetric criteria for three disease sites (head and neck, lung, and prostate) from the RTOG 0920, 1308, and 0938 protocols were adopted. We propose a simulated model for PTV dose-volume histograms (DVHs) of typical RT plans that incorporate the specified $D_{\min }$ and $D_{\max }$ values as variables. The DVHs were applied to the published TCP models to investigate the variations in TCP when the $D_{\min }$ is between $0 \%$ and $3.5 \%$ of the PTV and when the $D_{\max }$ is between $100 \%$ and $96.5 \%$ of the PTV. The effects of PTV changes on lung cancer were also studied. 


\section{Methods and Materials}

\subsection{Target DVH Models}

RTOG protocols are used to specify radiotherapy treatment plan quality criteria for clinical trials. Our study adopted the tumor target coverage criteria from the RTOG 0920 protocol for head and neck cancer, the RTOG 1308 protocol for non-small cell lung cancer (NSCLC), and the RTOG 0938 protocol for prostate cancer. The tumor volume dosimetry criteria for these three protocols are listed in Table 1, which include 1) the prescription dose coverage of D95\%; 2) $D_{\max }$ criteria that specify the maximum dose for the PTV; and 3) $D_{\min }$ criteria that specify the minimum dose for the PTV. As the DVH represents the cumulative coverage of a distributed dose obtained in individual PTV voxels, when such a dose is simulated by a truncated, skewed, Gaussian distribution, the DVH curve can be simulated to satisfy all three dosimetry criteria. The truncated points at the left and right two tails represent the $D_{\min }$ and $D_{\max }$ that the tumor receives, respectively. Figure 1 plots the simulated DVHs for the three disease sites.

Variations in the nominal DVH can be reconstructed when the defined $D_{\min }$ and $D_{\max }$ values deviate from the point dose. Here, the $D_{\max }$ deviation is defined

Table 1. PTV dosimetry criteria and TCP model parameters.

\begin{tabular}{|c|c|c|c|c|}
\hline & & $\begin{array}{l}\text { Head and Neck PTV } \\
\text { DVH RTOG } 0920\end{array}$ & $\begin{array}{l}\text { Lung PTV DVH } \\
\text { RTOG } 1308\end{array}$ & $\begin{array}{c}\text { Prostate PTV DVH } \\
\text { RTOG } 0938\end{array}$ \\
\hline \multicolumn{2}{|c|}{ Prescription dose } & $60 \mathrm{~Gy}$ & 70 Gy (2 Gy/fraction) & $51.6 \mathrm{~Gy}$ (4.3 Gy/fraction) \\
\hline \multicolumn{2}{|c|}{$D_{\max }$ criteria } & 70 Gy & $84 \mathrm{~Gy}$ & $55.21 \mathrm{~Gy}$ \\
\hline \multicolumn{2}{|c|}{$D_{\min }$ criteria } & $56 \mathrm{~Gy}$ & $59.5 \mathrm{~Gy}$ & $49.05 \mathrm{~Gy}$ \\
\hline \multirow{2}{*}{$\begin{array}{l}\text { TCP model } \\
\text { parameters }\end{array}$} & D50 & $51.77 \mathrm{~Gy}$ & $84.5 \mathrm{~Gy}$ & $70.5 \mathrm{~Gy}$ \\
\hline & $\gamma 50$ & 2.28 & 1.5 & 2.9 \\
\hline
\end{tabular}
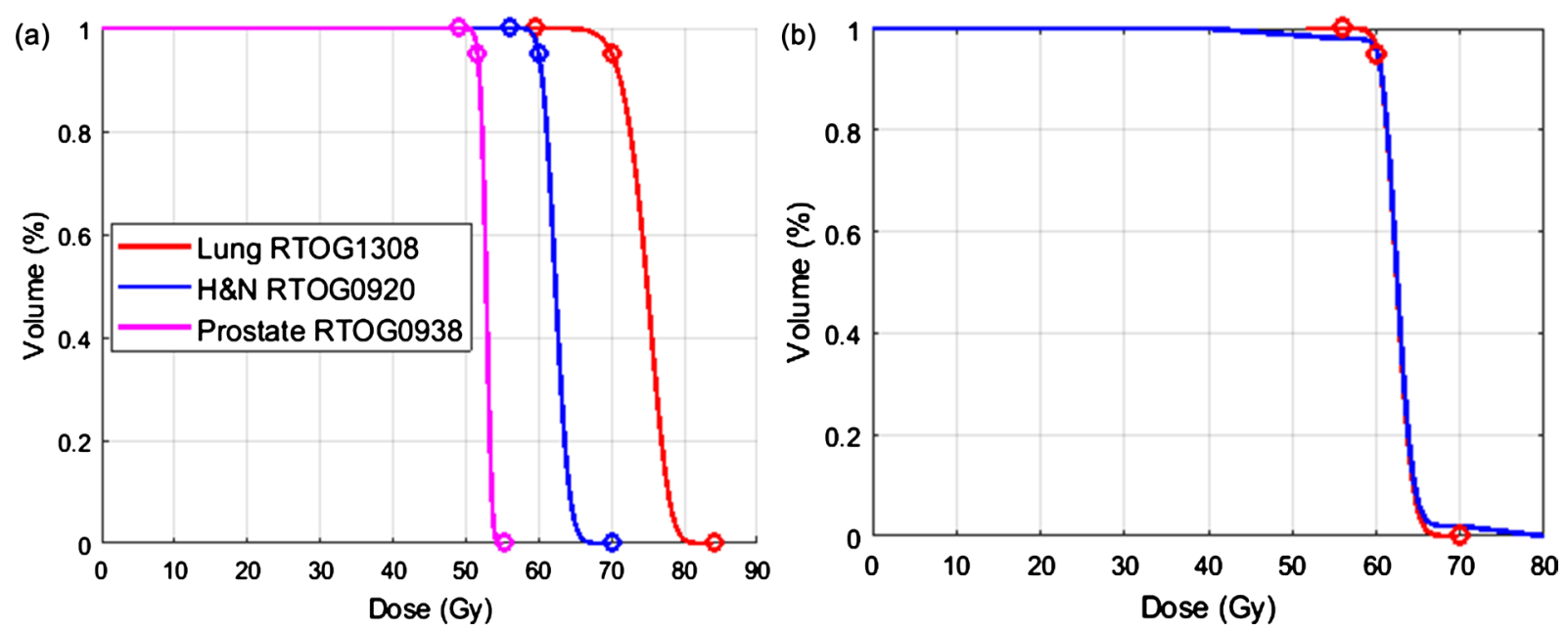

Figure 1. (a) The simulated nominal DVHs that satisfy the dosimetric criteria from the RTOG 0920, 1308, and 0938 protocols. The circles represent the prescription dose and the $D_{\min }$ and $D_{\max }$ criteria from the protocols. (b) A reconstructed DVH (blue) with deviations in the $D_{\min }$ and $D_{\max }$ values from the nominal DVH (red) for the RTOG 0920 protocol. Shown here is the $D_{\max }$ at $1 \%(\eta=1)$ and the $D_{\min }$ at $99 \%(\delta=1)$ for $40 \mathrm{~Gy} \leq \mathrm{PTV}$ dose $\leq 80 \mathrm{~Gy}$. 
as $\eta \%$ of the PTV, and the $D_{\min }$ deviation is defined as $(100-\delta) \%$ of the PTV. In this study, we investigated the variations in the $D_{\min }$ and $D_{\max }$ values up to $3.5 \%$ of the PTV, that is, $\eta$ and $\delta$ variations from 0 to 3.5. The actual $D_{\min }$ and $D_{\max }$ values of the entire PTV were considered additional variables that were assumed to vary from 5 to $30 \mathrm{~Gy}$ from the defined $D_{\min }$ and $D_{\max }$ criteria.

\subsection{TCP Models for the Three RTOG Protocols}

To examine the effects of changes in the PTV DVH coverage, we employed published TCP models. RTOG 0920 is a phase III study of postoperative RT for locally advanced resected head and neck cancers, with a prescription dose of $60 \mathrm{~Gy}$ and $D_{\min }$ and $D_{\max }$ values of $70 \mathrm{~Gy}$ and $56 \mathrm{~Gy}$, respectively. Okunieff et al. [8] published a TCP model with local control 50\% dose $\left(\mathrm{D}_{50}\right)$ and $\gamma_{50}$ as the change in TCP when a $1 \%$ change in dose around $\mathrm{D}_{50}$ occurs:

$$
\mathrm{TCP}=\exp \left[4 \gamma_{50}\left(\frac{D}{D_{50}}-1\right)\right] /\left\{1+\exp \left[4 \gamma_{50}\left(\frac{D}{D_{50}}-1\right)\right]\right\} .
$$

For head and neck cancer, the $D_{50}$ and $\gamma_{50}$ used were $51.77 \mathrm{~Gy}$ and 2.28, respectively.

RTOG 1308 is a phase III randomized trial comparing overall survival after either photon chemoradiotherapy or proton chemoradiotherapy for inoperable stage II-IIIB NSCLC. The target volume dosimetry review criteria are as follows: the prescription dose for PTV is $70 \mathrm{~Gy}$, the $D_{\max }$ should not exceed $84 \mathrm{~Gy}$, and the $D_{\text {min }}$ should not drop below 59.5 Gy (Table 1 ). The TCP model derived from the logistic expression was used for the calculation [9]:

$$
\operatorname{TCP}\left(\left\{D_{i}, v_{i}\right\}\right)=\prod_{i=1}^{N}\left[\frac{1}{1+\left(\frac{D_{50}}{D_{i}}\right)^{4 \gamma_{50}}}\right]^{v_{i}} \text {, where } \sum_{i=1}^{N} v_{i}=1 .
$$

The parameters for this model were obtained from a study by Martel et al. in 1999 [10] on local progression-free survival at 30 months, where $D_{50}$ and $\gamma_{50}$ were 84.5 Gy and 1.5, respectively (Table 1 ). To further study the volume effects on TCP, the Fenwick [11] and Martel models were used in a side-by-side comparison using 200-, 400-, and 600-cc tumor volumes having similar DVH curves:

$$
\mathrm{TCP}=\phi\left(\frac{D-D_{50}-c(\ln V-5)}{m D}\right)
$$

where $D_{50}=84.6 \mathrm{~Gy}, m=0.329, c=9.58, V$ is the volume in $c c$, and $\varphi$ is a Gaussian integral.

RTOG 0938 is a randomized phase II trial of hypofractionated radiotherapy for favorable-risk prostate cancer. In one of the two treatment legs, the prescription dose for PTV was $51.6 \mathrm{~Gy}$ in $4.3 \mathrm{~Gy}$ fractions; the $D_{\max }$ (no more than 0.03 cc of the PTV as defined by the RTOG protocol) should not exceed $55.21 \mathrm{~Gy}$, and the $D_{\min }$ (no more than $0.03 \mathrm{cc}$ of the PTV as defined by the RTOG proto- 
col) should not drop below 49.05 Gy (Table 1). The same TCP formula, as shown in Equation (1), was used, with parameters from a study by Levegrun et al. [11] (Table 1).

The differential DVH where a dose and corresponding volume fraction of the PTV was derived for a given DVH curve from the DVH model described above. The values were utilized in the corresponding disease site TCP models to obtain the volumetric average TCP for a given DVH curve. For each disease site, the TCP was first calculated with the modeled nominal DVHs. The impact of the $D_{\min }$ and $D_{\max }$ variations was assessed using the calculated TCPs from the different DVHs.

\section{Results}

Using a truncated, skewed, Gaussian distribution, the nominal DVHs that satisfy all three RTOG protocols can be simulated to satisfy the specified PTV volume dosimetry criteria. The truncated tails represent the $D_{\min } / D_{\max }$ point doses. These results are shown in Figure 1(a). Figure 1(b) shows the reconstructed DVH with the defined $D_{\max }$ at $1 \%(\eta=1)$ and the defined $D_{\min }$ at $99 \%(\delta=1)$ of the tumor volume, where the PTV dose covering the entire volume is greater than or equal to $40 \mathrm{~Gy}$ and less than or equal to $80 \mathrm{~Gy}$. This DVH maintains the prescription dose coverage as the nominal DVH.

The nominal and altered DVHs with the given $\eta$ and $\delta$ values were used to calculate the volumetric average TCP. The differences were examined as a function of $\eta$ and $\delta$, where $\eta$ and $\delta$ vary from 0 to 3.5. Figure 2(a) and Figure 2(b) show the variations in TCP for head and neck cancer and prostate cancer caused by deviations in the $D_{\min }$ and $D_{\max }$ from the RTOG 0920 and 0938 protocols using the TCP models described by Okunieff et al. [9] and Levegrun et al. [11], respectively. The head and neck TCP was found to vary from $-3.7 \%$ to $0.4 \%$ with the $D_{\min }$ and $D_{\max }$ deviations from the nominal TCP of $86.5 \%$ using the RTOG 0920 criteria. The prostate TCP was found to vary from the nominal TCP of $94.2 \%$, and a large variation of $-3.3 \%$ occurred when the $D_{\min }$ deviated from the criteria. Figure 2(c) shows the variations in the lung TCP from the nominal value of $32.4 \%$ using the Martel model. The TCP value is reduced by $-2.2 \%$ when the $D_{\min }$ is $96.5 \%$ of the PTV volume. When the volume of the PTV is considered in the Fenwick model, the change in TCP varies with tumor size. In our sampling of patients with stage II-IIIB NSCLC, the lung PTV was determined to be $378 \pm 196 \mathrm{cc}(\mathrm{n}=32)$. We selected tumor sizes of $200 \mathrm{cc}, 400 \mathrm{cc}$, and $600 \mathrm{cc}$ using the Fenwick model to study lung TCP variations with $D_{\min }$ and $D_{\max }$ deviations, as shown in Figures 2(d)-(f). At smaller volumes (e.g., $200 \mathrm{cc}$ ), the nominal TCP is larger (54.0\%) at the same prescription dose but is reduced to $35.7 \%$ and $23.1 \%$ at larger volumes of $400 \mathrm{cc}$ and $600 \mathrm{cc}$, respectively. The variations in TCP due to the $D_{\max }$ definition of $\eta=3.5 \%$ were $2.4 \%, 2.8 \%$, and $2.8 \%$, but was less than $-0.3 \%$ from the $D_{\min }$ defined as $(100-\delta) \%$ of the volume. The TCP variations with $\eta$ or $\delta$ equaling $1 \%, 2 \%$, or $3 \%$ in these models at the three 
disease sites are listed in Table 2. The TCP values for head and neck cancer and prostate cancer, but not lung cancer, are negatively affected by deviations in $D_{\min }$. The lung TCP value increased to a greater extent when the $D_{\max }$ value varied

(a)

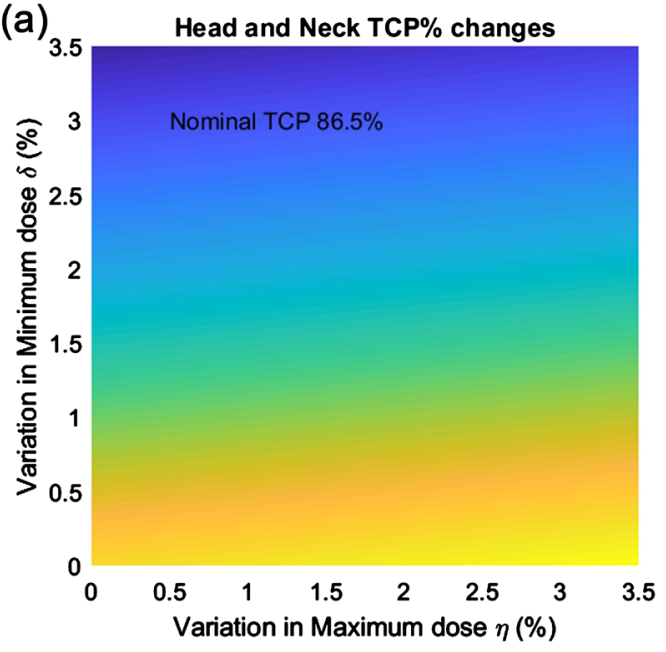

(C) $3.5 \quad$ Lung TCP\% changes (Martel)
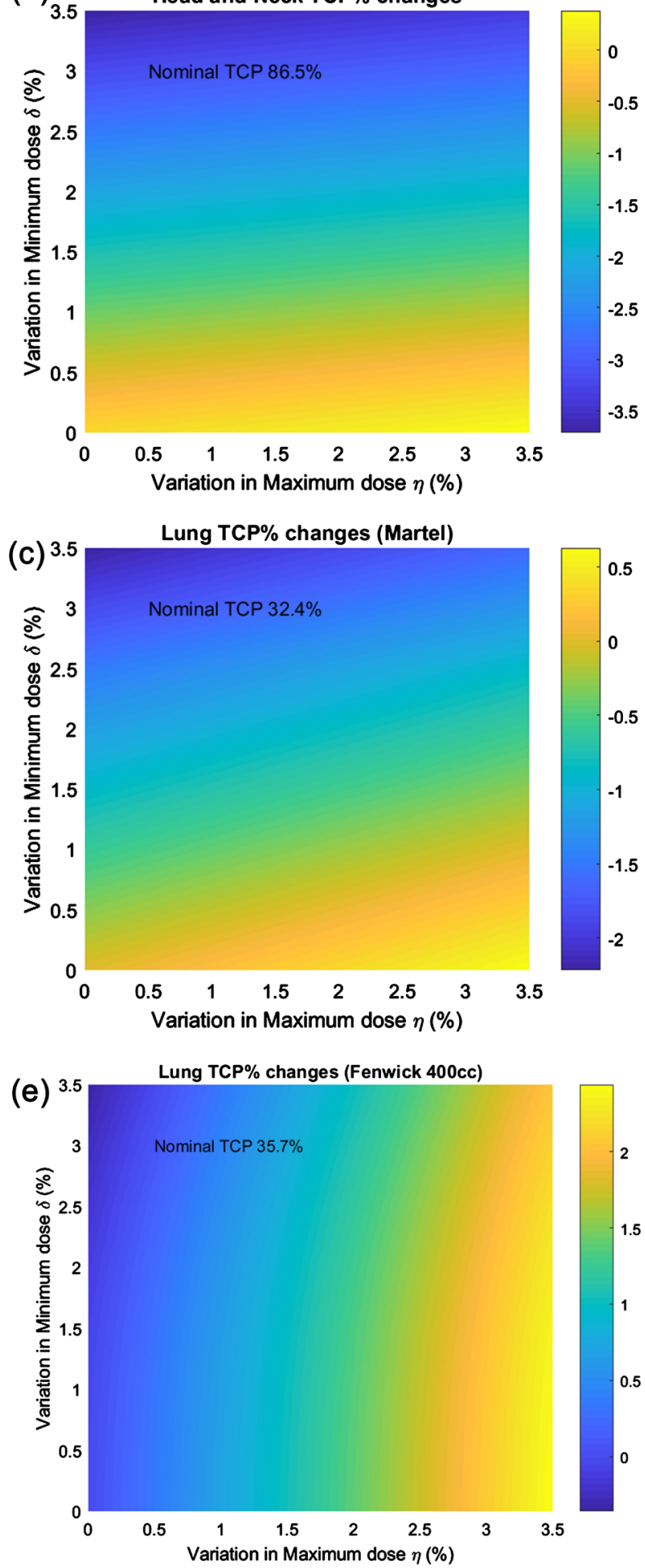

(b)
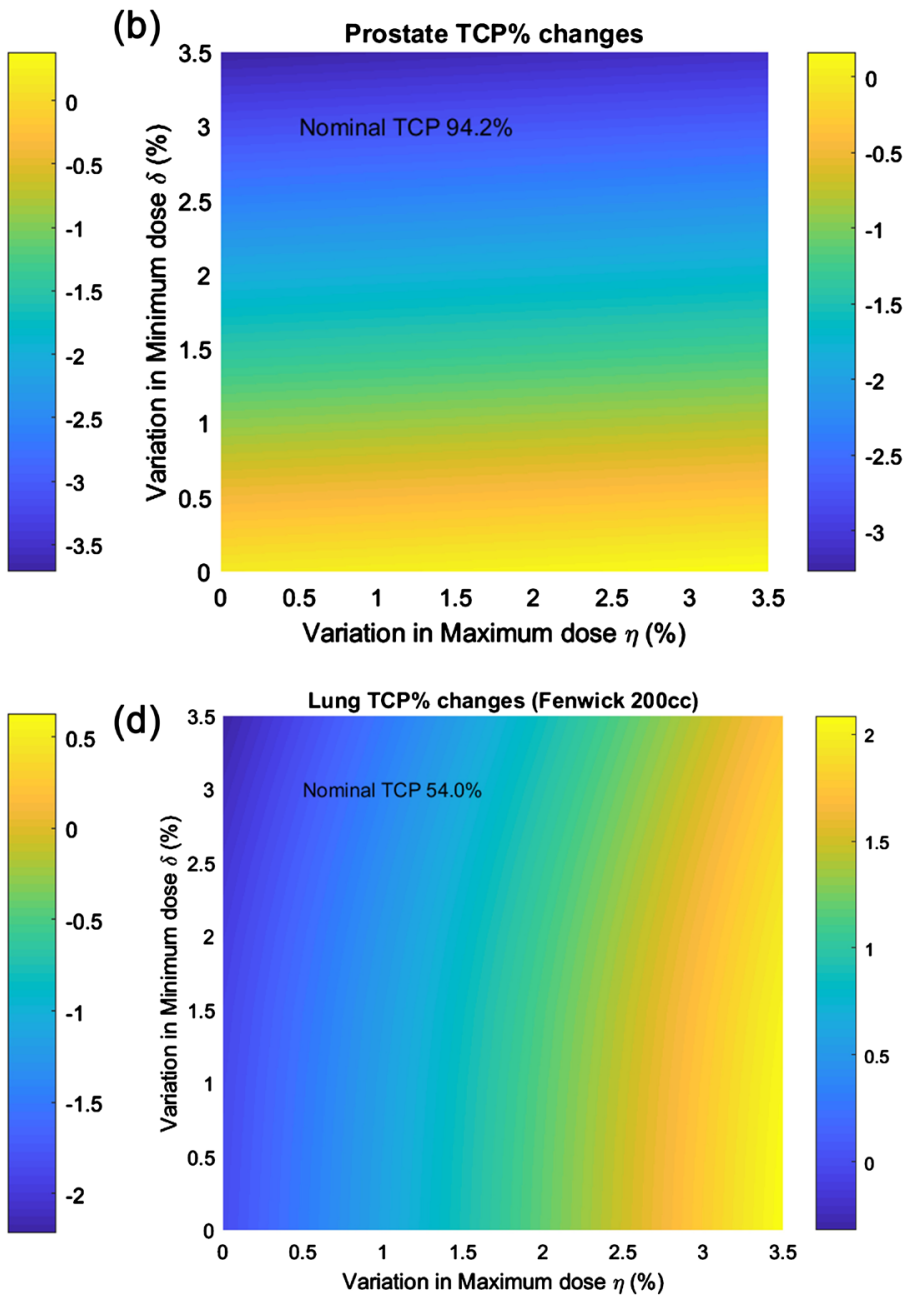

(f)

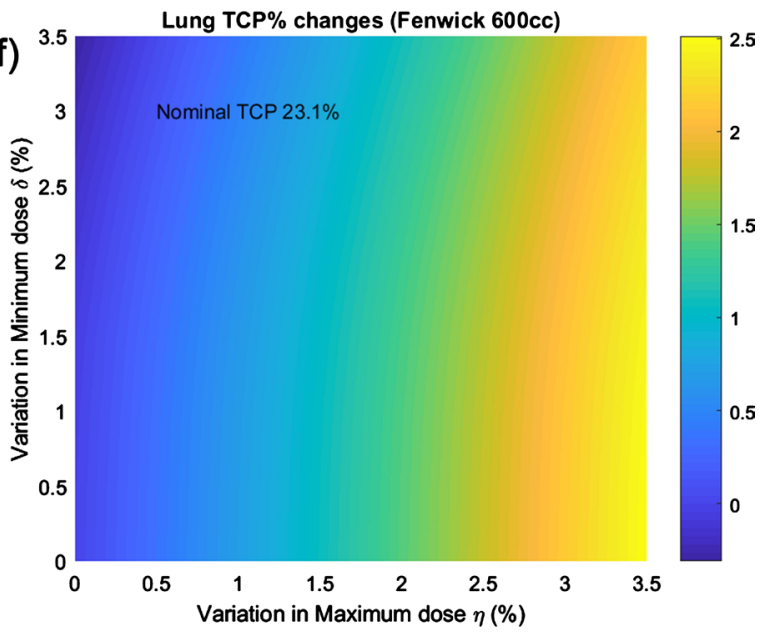

Figure 2. Two-dimensional plots of TCP variations from head and neck, prostate, and lung models due to variations from the defined $D_{\min } / D_{\max }$ values of $(100-\delta) \%$ and $\eta \%$ of the PTV. 
Table 2. Modeled TCP variations from nominal values with $\eta$ or $\delta$ equaling $1 \%$, $2 \%$, or $3 \%$ using dosimetric criteria from the RTOG 0920, 1308, and 0938 protocols.

\begin{tabular}{ccccccc}
\hline & $\begin{array}{c}\text { Head and } \\
\text { Neck }\end{array}$ & Prostate & $\begin{array}{c}\text { Lung } \\
\text { Martel }\end{array}$ & $\begin{array}{c}\text { Lung } \\
\text { Fenwick } \\
200 \mathrm{cc}\end{array}$ & $\begin{array}{c}\text { Lung } \\
\text { Fenwick } \\
\mathbf{4 0 0} \mathrm{cc}\end{array}$ & $\begin{array}{c}\text { Lung } \\
\text { Fenwick } \\
\mathbf{6 0 0} \mathrm{cc}\end{array}$ \\
\hline Nominal TCP $(\%)$ & 86.5 & 94.2 & 32.4 & 54.2 & 35.7 & 23.1 \\
min dose @ 99\% $(\delta=1)$ & -1.1 & -0.9 & -0.6 & 0.0 & 0.0 & 0.0 \\
min dose @ 98\% $(\delta=2)$ & -2.1 & -1.9 & -1.2 & -0.1 & -0.1 & -0.1 \\
$\min$ dose @ 97\% $(\delta=3)$ & -3.2 & -2.8 & -1.9 & -0.2 & -0.3 & -0.2 \\
max dose @ 1\% $(\eta=1)$ & 0.1 & 0.0 & 0.2 & 0.6 & 0.7 & 0.7 \\
$\max$ dose @ 2\% $(\eta=2)$ & 0.2 & 0.1 & 0.4 & 1.2 & 1.4 & 1.4 \\
$\max$ dose @ 3\% $(\eta=3)$ & 0.3 & 0.1 & 0.5 & 1.8 & 2.1 & 2.1 \\
\hline
\end{tabular}

from the head and neck and prostate targets.

\section{Discussion}

The TCP calculations performed in this study were all based on the simulated target DVHs that assume that the dose distribution in the target is a skewed Gaussian distribution and fitted to the criteria from the RTOG protocols. Separate published TCP model parameters at three different disease sites were used to study the TCP variations of different specifications of $D_{\max }$ and $D_{\min }$ evaluations of PTV. TCP was observed to vary as the relative volume definitions of $D_{\max }$ and $D_{\min }$ changed, according to the results shown in Table 2; a deviation in $D_{\max }$ up to $3 \%$ of the volume did not result in an increase in the TCP $(\leq 2.1 \%)$ during the radiotherapy treatment of lung, head and neck, and prostate cancers. This finding is because slightly greater target volumes receive a higher dose of RT. When the $D_{\min }$ definition deviated from the point dose, a greater volume received less than the $D_{\min }$ value, and the TCP was likewise reduced. For head and neck and prostate cancers, the reduction in TCP can approach $-3.2 \%$ and $-2.8 \%$, respectively, when the $D_{\min }$ is defined as $97 \%$ of the volume. If one limits the $D_{\min }$ deviation to $97 \%$, the TCP will decrease by no more than $-1 \%$ using either the Martel or Fenwick model. In our sampling of patients using RTOG protocols, the PTV volumes was evaluated to be $378 \pm 196 \mathrm{cc}$ (lung, $\mathrm{n}=32$ ), 353 $\pm 230 \mathrm{cc}$ (head and neck, $\mathrm{n}=73$ ), and $95 \pm 32 \mathrm{cc}$ (prostate, $\mathrm{n}=148$ ). Therefore, when the $D_{\min }$ and $D_{\max }$ values are defined as $0.03 \mathrm{cc}$ of the PTV as in the current RTOG protocols [6], $0.03 \mathrm{cc}$ as a negligible percentage of tumor volume will correspond to negligible TCP variations for lung, head and neck, and prostate volumes. If one limits the TCP variations within $2.1 \%$, the definition of the $D_{\min }$ should be kept at $98 \%$ for patients with head and neck and prostate cancer, but relaxed to $97 \%$ for lung cancer, whereas D3\% can be used as the $D_{\max }$.

Although a thorough literature search was performed for TCP models and related parameters used in this study, we acknowledge that the calculated TCP values only provide very rough predictions. Further studies to incorporate bio- 
logical theories and more practical empirical modeling [12] of predictions of tumor control are ongoing. We also recognize that the assumptions implicit in skewed Gaussian distributions are not realistic for all patient plans. However, despite these rough predictions, we believe that the model calculations used in this study can still be applied to elucidate meaningful dosimetric parameters for evaluations of radiotherapy plan quality.

\section{Conclusions}

Our study investigated the effects on TCP by deviations in the $D_{\min }$ and $D_{\max }$ values up to $3.5 \%$ of the tumor volume for head and neck, lung, and prostate cancer patients, using published TCP models and parameters. The results of this study can be used for plan quality evaluations when the $D_{\min }$ and $D_{\max }$ values slightly deviate from the point dose. When the overall target prescription dose coverage is maintained, it is recommended that the $D_{\max }$ be within $3 \%$ of the PTV: $98 \%$ (for head and neck and prostate) and 97\% (for lung) of the target volume, satisfying the $D_{\min }$ to maintain TCP variations at less than $2.1 \%$. Using $0.03 \mathrm{cc}$ instead of the point dose for $D_{\min }$ and $D_{\max }$ values at all three disease sites minimally impacts TCPs.

One drawback of this study is that the conclusion is made solely based on simulations. There is no consensus on TCP models; therefore, two models were selected to compare the results. In the future, more clinical data from the above-mentioned clinical trials will be available with patient outcome. Tumor control probability should be evaluated with real patient dose distributions to make the conclusions from this research more acceptable to clinical practices.

This work is funded by NCI for clinical trial data quality assurance (QA). The research outcome of this work directly impacts the daily QA workflow performed by IROC.

\section{Funding}

This project was supported by grants U10CA180868 and U24CA180803 from the National Cancer Institute.

\section{Conflicts of Interest}

The authors declare no conflicts of interest regarding the publication of this paper.

\section{References}

[1] Bekelman, J.E., Deye, J.A., Vikram, B., et al. (2012) Redesigning Radiotherapy Quality Assurance: Opportunities to Develop an Efficient, Evidence-Based System to Support Clinical Trials-Report of the National Cancer Institute Work Group on Radiotherapy Quality Assurance. International Journal of Radiation Oncology • Biology • Physics, 83, 782-790. https://doi.org/10.1016/j.ijrobp.2011.12.080

[2] Purdy, J.A. (2008) Quality Assurance Issues in Conducting Multi-Institutional Advanced Technology Clinical Trials. International Journal of Radiation Oncology • 
Biology • Physics, 71, S66-S70. https://doi.org/10.1016/j.ijrobp.2007.07.2393

[3] Palta, J.R., Deye, J.A., Ibbott, G.S., et al. (2004) Credentialing of Institutions for IMRT in Clinical Trials. International Journal of Radiation Oncology • Biology • Physics, 59, 1257-1259. https://doi.org/10.1016/j.ijrobp.2004.03.007

[4] Fitz Gerald, T.J. (2014) A New Model for Imaging and Radiation Therapy Quality Assurance in the National Clinical Trials Network of the National Cancer Institute. International Journal of Radiation Oncology • Biology • Physics, 88, 272-273. https://doi.org/10.1016/j.ijrobp.2013.09.030

[5] Carrie, C., Hoffstetter, S., Gomez, F., et al. (1999) Impact of Targeting Deviations on Outcome in Medulloblastoma: Study of the French Society of Pediatric Oncology (SFOP). International Journal of Radiation Oncology • Biology • Physics, 45, 435-439. https://doi.org/10.1016/S0360-3016(99)00200-X

[6] NRG Oncology Center for Innovation Website. https://www.nrgoncology.org/Clinical-Trials

[7] Grégoire, V. and Mackie, T.R. (2011) State of the Art on Dose Prescription, Reporting and Recording in Intensity-Modulated Radiation Therapy (ICRU Report No. 83). Cancer Radiotherapie, 15, 555-559. https://doi.org/10.1016/j.canrad.2011.04.003

[8] Okunieff, P., Morgan, D., Niemierko, A., et al. (1995) Radiation Dose-Response of Human Tumors. International Journal of Radiation Oncology • Biology • Physics, 32, 1227-1237. https://doi.org/10.1016/0360-3016(94)00475-Z

[9] Suit, H., Shalek, R. and Wette, R. (1965) Cellular Radiation Biology. 514-530.

[10] Martel, M.K., Ten Haken, R.K., Hazuka, M.B., et al. (1999) Estimation of Tumor Control Probability Model Parameters from 3-D Dose Distributions of Non-Small Cell Lung Cancer Patients. Lung Cancer, 24, 31-37. https://doi.org/10.1016/S0169-5002(99)00019-7

[11] Fenwick, J.D., Nahum, A.E., Malik, Z.I., et al. (2009) Escalation and Intensification of Radiotherapy for Stage III Non-Small Cell Lung Cancer: Opportunities for Treatment Improvement. Clinical Oncology, 21, 343-360. https://doi.org/10.1016/j.clon.2008.12.011

[12] Levegrün, S., Jackson, A., Zelefsky, M.J., et al. (2002) Risk Group Dependence of Dose-Response for Biopsy Outcome after Three-Dimensional Conformal Radiation Therapy of Prostate Cancer. Radiotherapy and Oncology, 63, 11-26. https://doi.org/10.1016/S0167-8140(02)00062-2 